\title{
Visualització del moviment harmònic simple aplicant les noves tecnologies
}

\author{
Ernest Arnau Marco (adjunts@gmail.com) I.E.S Pascual Carrión, (Sax, Espanya)
}

En el present article es descriuen diversos mètodes per estudiar el moviment harmònic simple (M.H.S) d'una molla i el corresponent a un punt en el moviment circular. A l'inici es realitza una deducció de les equacions per, a continuació, procedir a la seva comprovació per mitjà de dues vies: mesures directes de l'experiment i valors obtinguts amb ajuda de programari informàtic. Els programes que utilitzem exploten la capacitat de les noves tecnologies disponibles: el PC i el mòbil.

Paraules clau: moviment harmònic simple, molla, "tracker", physics toolbox, experiment, noves tecnologies, vídeo, mòbil, llei de Hooke.

In this article several methods are described for the study of the harmonic simple movement (M.H.S) of a metallic spring and the movement of a certain point in the circle movement. First, a deduction of the equations is done in order to prove them by means of two different methods: direct measures from the experiment and obtained values from the software. The programs that have been used are based on the capacities of new available technologies such as: the pc and mobile phones.

Keywords: harmonic simple movement, metallic spring, "Tracker”, "Physics Toolbox", experiment, new technologies, video, mobile phone, Hooke's law.

\section{INTRODUCCIó}

En l'actualitat, el docent compta amb recursos que fa un parell de dècades eren inimaginables. Des de fa uns anys tenim la possibilitat de gravar el moviment $\mathrm{i}$ analitzar-lo a l'instant amb els nostres alumnes. Aquest és un dels nostres objectius: utilitzar les possibilitats que ens ofereixen les noves tecnologies per estudiar el moviment d'un cos, subjecte a una molla i el corresponent a un moviment circular.

El moviment harmònic simple (M.H.S) és un dels temes que més ens trobem en física i, per tant, en la natura. Això es deu a què podem aproximar gran quantitat de sistemes físics a un M.H.S. La importància d'aquest moviment ve contemplada en el temari de primer i segon de batxillerat al incloure el M.H.S en un dels blocs d'estudi. Al llarg dels cursos acadèmics s'han observat problemes en un gran nombre d'alumnes de diferents promocions en la resolució d'aquesta part de la física.
Quan procedim a analitzar les causes de dita dificultat ens trobem que són incapaços de visualitzar com és en realitat el moviment que estem analitzant.

Junt a la visualització de gravacions dels experiments realitzats a l'aula tenim la possibilitat d'utilitzar multitud d'aplicacions disponibles tant per a mòbils com ordinadors. En el nostre cas utilitzarem el programa "Tracker" (Linux, Windows i Mac) i l'aplicació per a mòbil "Physics Toolbox" (disponible per a Android i iOS); ambdues gratuïtes.

\section{FONAMENTACIÓ TEÒRICA}

\section{Deducció de les equacions per al M.H.S en una molla}

Al penjar d'una molla (amb constant $\mathrm{k}$ ) una peça de massa $\mathrm{m}$ i desplaçar-la de la seva posició d'equi- 
libri, observem un moviment periòdic. La mera contemplació de la peça ens indueix a trobar una equació amb solució sinusoïdal (periòdica en el temps). Si situem l'origen de coordenades a la posició d'equilibri i, al nivell de batxillerat, no tenim en compte la influència del pes, podem deduir que:

$\left.\begin{array}{l}F=-k \cdot y \\ F=m \cdot a_{y}\end{array}\right) \rightarrow m \cdot a_{y}=-k \cdot y \rightarrow a_{y}=-\frac{k}{m} y \rightarrow \frac{d^{2} y}{d^{2}}+\frac{k}{m} y=0$

Què és una equació diferencial amb solució:

$$
\mathrm{y}=\mathrm{A} \cdot \sin \left(\sqrt{\frac{\mathrm{k}}{\mathrm{m}}} \cdot \mathrm{t}+\emptyset\right)
$$

En la part "Anàlisis dels resultats" del present article compararem l'equació anterior amb l'obtinguda directament de la gràfica del moviment, on $\omega$ és la freqüència pròpia de l'oscil·lador que es troba relacionada amb el període de la forma.

$$
\omega=\frac{2 \pi}{\mathrm{t}} \rightarrow T=2 \pi \sqrt{\frac{m}{k}}
$$

Aquesta expressió també la comprovarem en la part final de l'article.

\section{VALORS EXPERIMENTALS PER AL M.H.S D'UNA MOLLA}

\section{La constant de la molla i la massa utilitzada}

En el punt anterior s'ha mostrat la forma "comú" d'introduir el moviment d'un cos subjecte a una molla. Considerem que explicar d'aquesta forma la física és quedar-se sense la bellesa que ens proporciona la natura. Adoptant la premissa de realitzar el major nombre de comprovacions experimentals, calcularem la constant de la molla per després obtenir la massa de la peça que penjarem en el M.H.S. El procediment experimental per calcular la constant s'explica en quart d'ESO, però és convenient realitzar un repàs abans d'aprofundir.

Amb l'elongació de la molla obtinguda després de penjar una peça de referència (Figura 1) procedirem a calcular dita constant. Tenint el valor de la constant, obtindrem la massa de la peça que utilitzarem en el M.H.S i comprovarem el nostre resultat amb la balança.

$K \rightarrow|\vec{F}|=|\vec{P}| \rightarrow k \Delta y=m \cdot g \rightarrow \mathrm{k}=\frac{m \cdot g}{\Delta y}=\frac{0,059,8}{0,17}=2,88 \frac{N}{m}$
Massa $\rightarrow|\vec{F}|=|\vec{P}| \rightarrow k \cdot \Delta y=m \cdot g \rightarrow m=\frac{k \Delta y}{g}=\frac{2,880,31}{9,8}=0,091 \mathrm{~kg}$

Aquest últim valor s'ajusta al valor mesurat a la balança que podem veure en la següent figura:
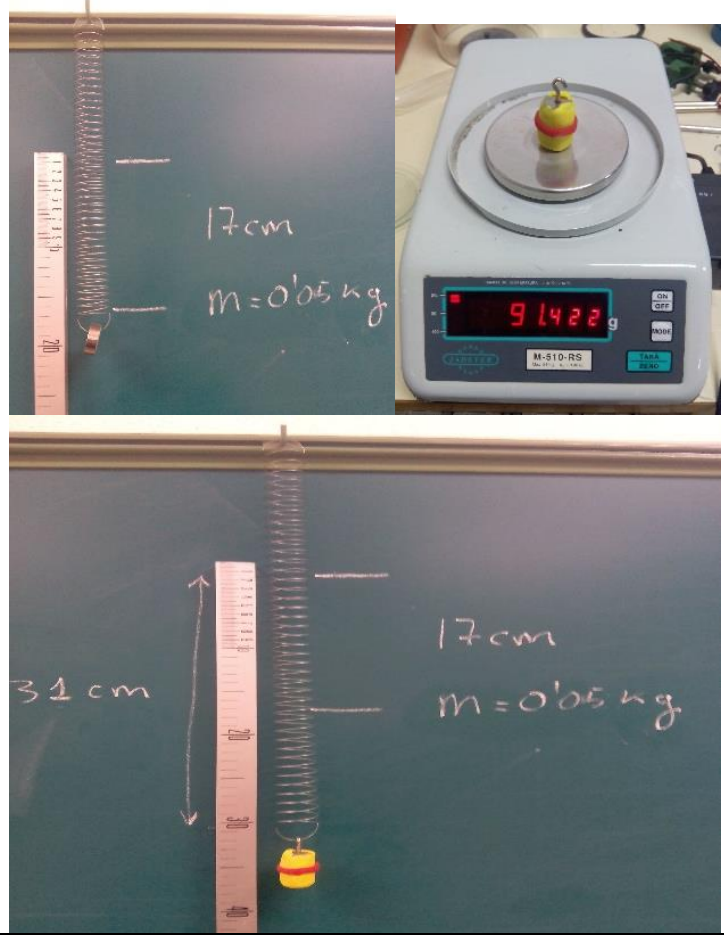

Figura 1. Elongació de la molla en funció de la massa que hi penja.

Com podem veure, la nostra molla amb la peça de referència amb la massa marcada $(m=50 \mathrm{~g})$ s'estira $17 \mathrm{~cm}$. La molla amb la peça que utilitzarem als diferents apartats veiem que s'estira $31 \mathrm{~cm}$.

\section{El moviment amb l’aplicació "Physics Toolbox"}

Un dels programes que ens permet visualitzar directament el tipus de moviment que estem estudiant és l'aplicació per a mòbil "Physics Toolbox", disponible gratuïtament.

L'aplicació "Physics Toolbox" utilitza l'acceleròmetre dels mòbils actuals per obtenir valors que es van representant al moment. Així, podem visualitzar directament a la pantalla el tipus de moviment que realitza el nostre dispositiu. Un possible mètode d'utilització és penjar el nostre mòbil en la molla, l'estirem, polsem inici i veiem el tipus de moviment en la pantalla (figura 2). 
L'aplicació també ens permet exportar les dades en un format .csv que podem obrir amb Excel o libreoffice per representar el moviment (figura 3). En les gràfiques veiem que si agafem valors durant temps llargs observem l'amortiment en el moviment. Podrem plantejar als nostres alumnes el motiu o en què s'ha transformat l'energia cinètica i potencial.

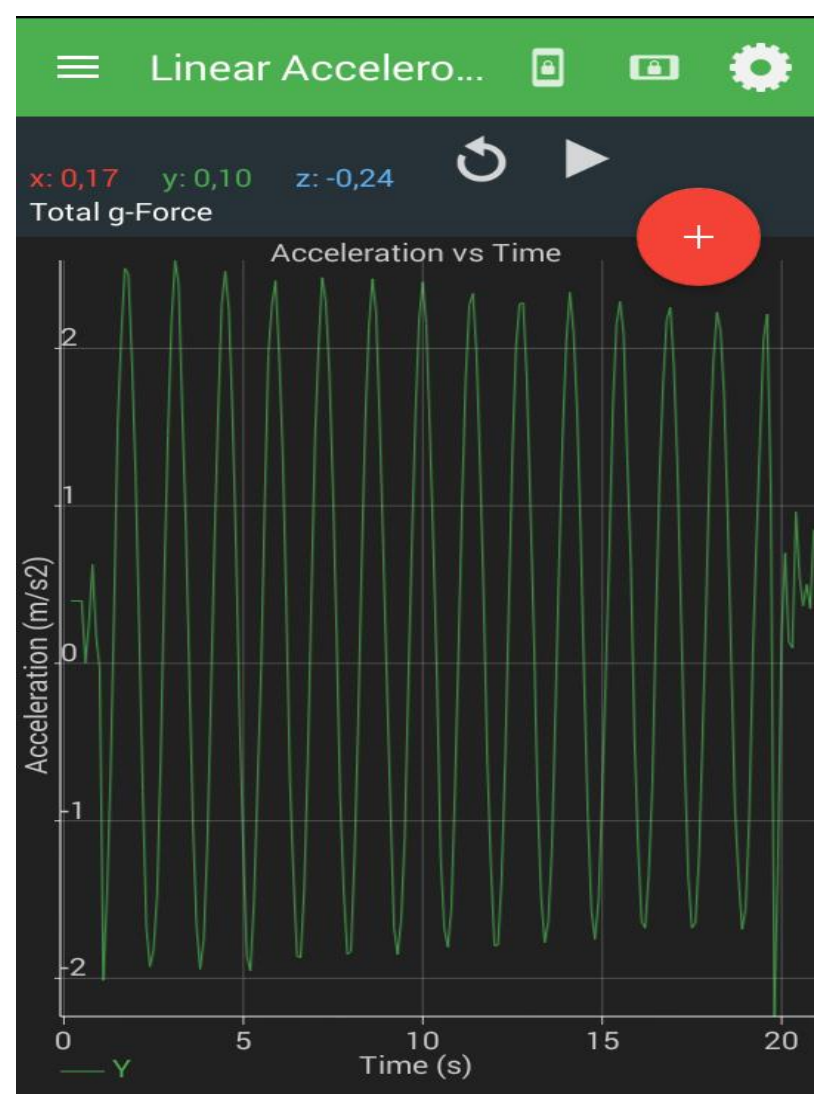

Figura 2. Captura de pantalla de l'aplicació "Physics Toolbox"; es pot apreciar l'amortiment de la molla.

Physics Toolbox

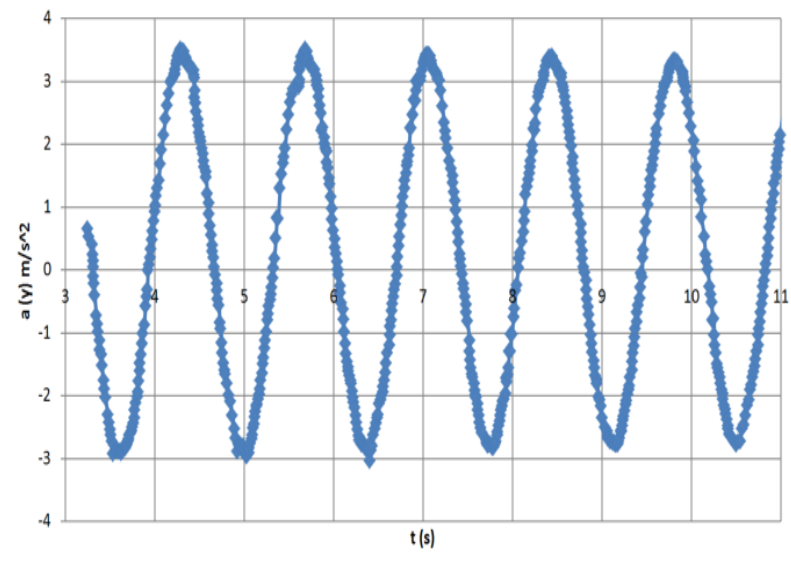

Figura 3. Representació d'Excel de l'acceleració obtinguda amb l'aplicació.

\section{Gravació i extracció de valors amb "Tracker"}

Per comprovar la validesa de les equacions teòriques hem d'obtenir els valors experimentals. A aquest efecte realitzarem l'enregistrament del moviment amb la càmera del mòbil per després, juntament amb els alumnes, analitzar el vídeo amb el programa "Tracker". Aquesta extracció de dades permetrà una major comprensió del moviment per part dels alumnes.

Per visualitzar millor la posició de la massa en el vídeo recobrim la peça de metall amb plastilina groga i li posem una tira de color vermell al centre (figura 1). El nostre enregistrament ha de ser el més estable possible; per reduir les vibracions recorrerem a deixar el mòbil sobre una taula a l'altura del M.H.S.

Durant la gravació mesurarem amb un cronòmetre el temps en el qual la peça realitza deu oscil-lacions (per minimitzar errors) obtenint un resultat de T10 $=11,1$ segons. D'aquesta forma, mirant la secció anàlisi dels resultats del present article, podrem comprovar el valor teòric donat per l'equació 2 amb el valor experimental. Cal destacar que podem obtenir el mateix valor amb la gravació que realitzem.

Obtinguda la gravació passem a l'anàlisi junt als nostres alumnes. El primer pas consistirà en conèixer el funcionament del software "Tracker". Executem el programa i importem el vídeo. Primer veurem la gravació per assegurar-nos que tenim una qualitat suficient per permetre localitzar en qualsevol moment els punts que ens interessen.

El programa "Tracker" permet definir el paràmetre "vara de calibratge" amb el qual, el propi programa, coneixerà les distàncies en el vídeo. (figura 3 , icona A). Amb aquest fi, a l'hora de gravar el vídeo hem posat una regla junt a la molla. El següent pas serà posar els eixos (figura 4, lletra B)Ja tenim a punt el programa per indicar-li la peça i extreure els punts. "Creem" una massa puntual (figura 4, lletra C) i definim "trajectòria automàtica". Per definir el punt (la nostra marca vermella sobre la plastilina groga) s'ha de polsar "ctrl" + "Majúscula" + "Botó esquerre".

A mesura que "Tracker" va agafant els punts observem, junt als alumnes, si aquests punts són correctes, així com la forma de la gràfica (figura 3 ). Tracker permet corregir els punts en el cas de ser incorrectes en la seva identificació. 


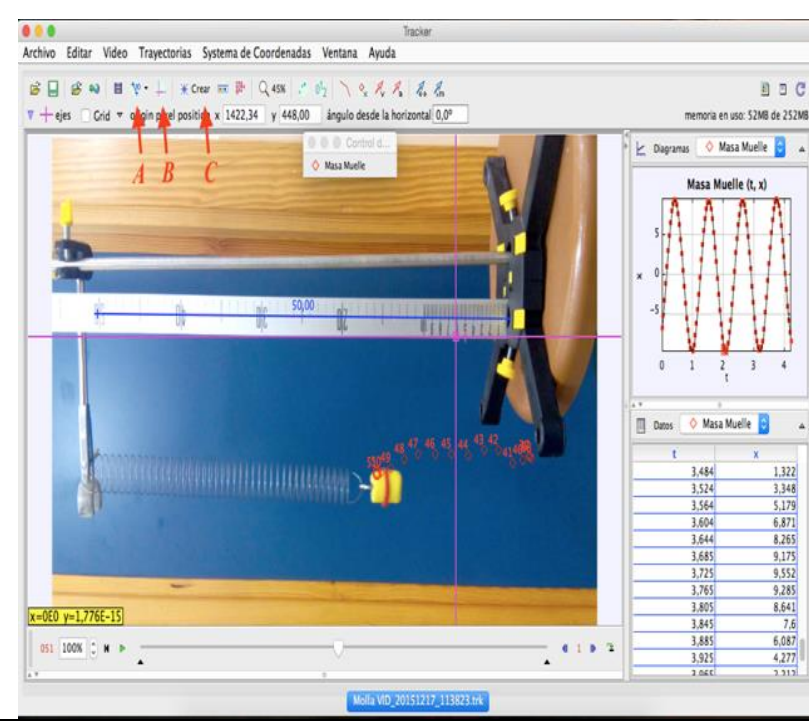

Figura 4. Funcionament del programa "Tracker". Les icones A, B i C indiquen la creació de la "vara de calibratge", "els eixos" i la " massa puntual".

Una vegada determinats els valors podem exportar-los per representar amb el full de càlcul (secció anàlisi de resultats) o veure directament la representació en el programa (figura 4). Veiem que la funció d'un moviment harmònic simple és sinusoïdal, podent ajustar-la a un sinus o a un cosinus. Així, amb les dades i les gràfiques tornem a calcular un període d'oscil-lació d'1,1 segons.

\section{EL M.H.S DUN MOVIMENT CIRCULAR}

Si abans de realitzar qualsevol explicació preguntem als nostres alumnes: "El moviment d'un punt en un disc que gira té relació amb el d'una peça que oscil la d'una molla?" El més probable és que no troben cap semblança. Aquest fet ens dóna una idea del poc intuïtiu que és el M.H.S. d'un moviment circular periòdic realitzat sense experiments.

Per tal d'esmenar aquesta falta d'intuïció ens disposem a utilitzar l'aplicació "Tracker" per veure el moviment. Pensem que no té sentit presentar als alumnes un moviment que no s'imaginen i, per tant, no entenen.

Procedirem d'igual forma que abans amb la moIla. Realitzarem una gravació, amb eixa anirem al programa "Tracker", identificarem els punts i el programa ens donarà la gràfica amb la qual podrem comprovar que és un M.H.S.

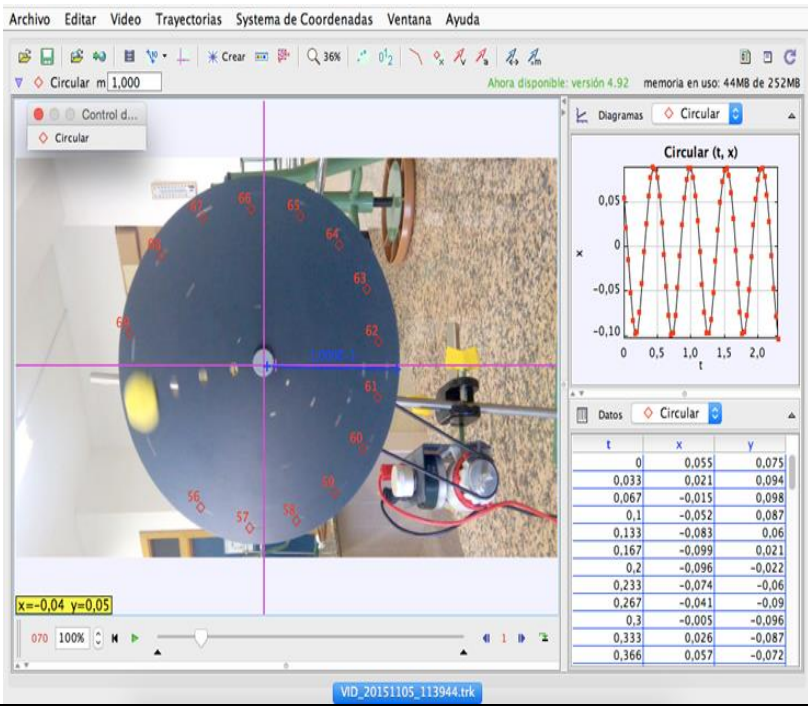

Figura 5. Anàlisi del moviment circular i comprovació, en la gràfica, del tipus de moviment que tenim.

\section{ANÀLISI DELS RESULTATS}

En aquesta part comprovarem la concordança dels valors calculats en la part teòrica, les mesures experimentals directes i les proporcionades per la gravació.

\section{Concordança entre equacions i moviment}

Les gràfiques obtingudes amb l'aplicació mòbil "Physics Toolbox" (figura 3 ) i amb el programa per ordinador "Tracker" (figures 6 i 7) mostren una distribució dels valors sinusoïdals tant per al cas d'una massa penjada en una molla com per a un moviment circular; fet que coincideix amb la forma deduïda en l'equació 1.

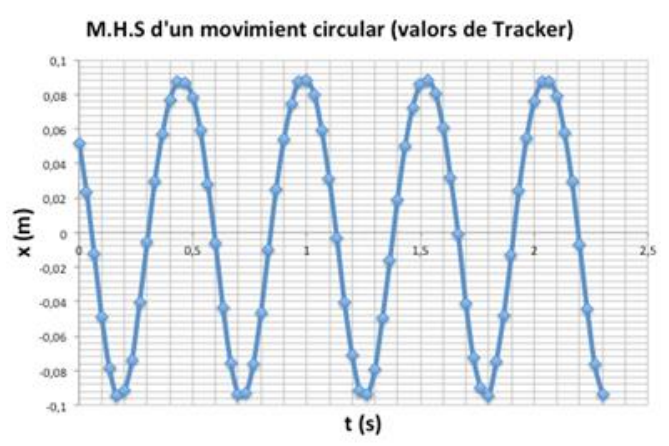

Figura 6. Gràfic M.H.S d'un moviment circular (valors de Tracker) realitzat amb Excel 


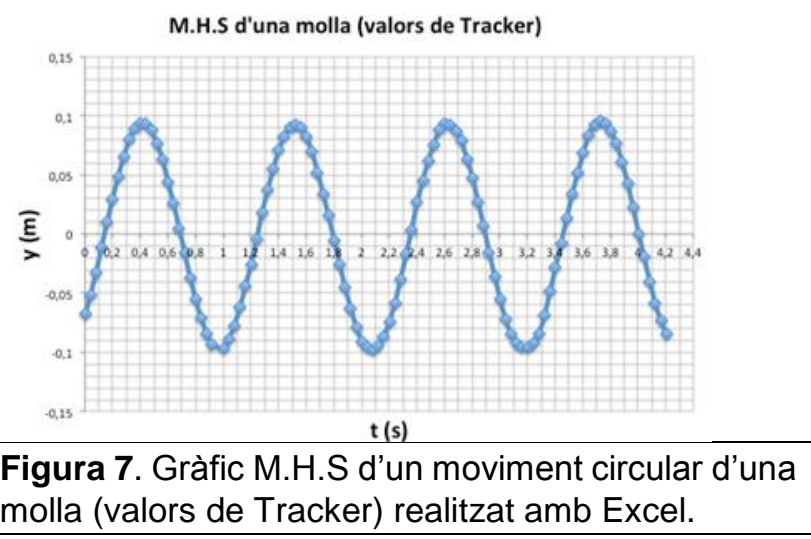

\section{El període}

Al llarg de la secció anterior s'ha obtingut experimentalment el valor de deu períodes del M.H.S T10 $=11,1$ segons. Valor que coincideix amb l'obtingut amb la visualització de la gravació i amb la gràfica dels punts (figura 6).

A continuació calcularem el valor teòric donat per l'equació 2 per a la massa calculada amb la llei de Hooke i el valor de la constant de la molla.

Valor teòric $\rightarrow T=2 \pi \cdot \sqrt{\frac{m}{k}}=2 \pi \sqrt{\frac{0,091}{2,88}}=1,12 \mathrm{~s}$

Valor experimental $\rightarrow T_{10}=11,1 \rightarrow T=1,11 \mathrm{~s}$

S'observa una gran concordança entre el valor teòric i l'experimental.

\section{Comprovació de les equacions}

El programa "Tracker", a través de la pestanya "ferramentes de dades", ens permet ajustar els punts a diferents funcions, entre elles el sinus. Utilitzarem aquesta característica per a comprovar l'ajust i comprovar el valor de l'equació general deduïda teòricament de l'equació 1.

En una primera aproximació veiem que l'estructura de l'equació teòrica i l'expressió que ajusta els valors coincideixen. El següent pas és comparar els valors esperats de la velocitat angular (calculats amb les equacions deduïdes al principi de l'article) amb els de l'ajust realitzat pel programa "Tracker" (figura 8).

$$
\text { Ajust de Tracker } \rightarrow \omega=5,686 \frac{\mathrm{rad}}{\mathrm{s}}
$$

Valor càlcul teòric $\rightarrow \omega=\sqrt{\frac{k}{m}}=\sqrt{\frac{2,88}{0,091}}=5,63 \frac{\mathrm{rad}}{\mathrm{s}}$

Podem observar un valor teòric $\mathrm{i}$ experimental molt pròxim, el que ens porta a donar per vàlida l'expressió deduïda per al M.H.S.

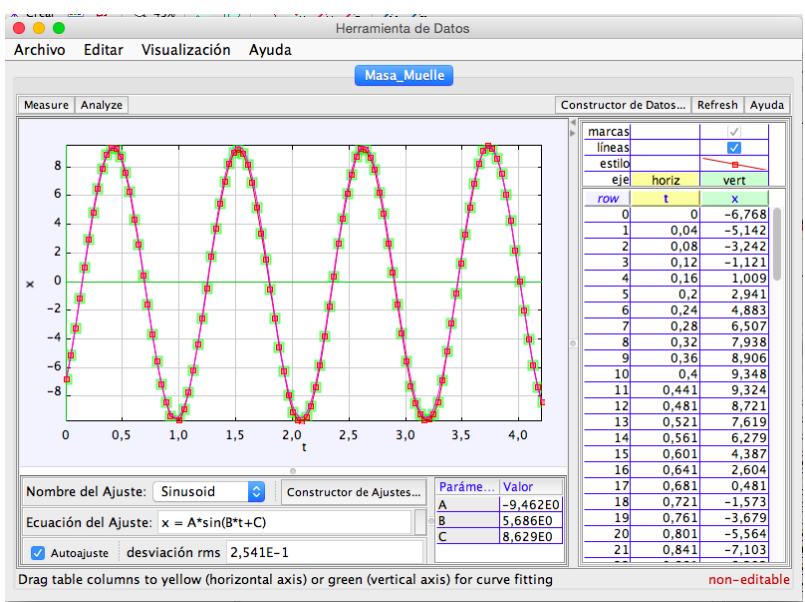

Figura 8. Gràfica representada pel programa "Tracker", es pot observar l'ajust sinusoïdal en la part inferior.

\section{CONCLUSIONS}

Després de la posada en pràctica de la presentació en classe de la teoria junt als experiments, es comprova un major grau de comprensió i assimilació de l'exposat. EI M.H.S deixa de tenir caràcter purament teòric per passar a moviments que els alumnes han observat, representat i comprovat. A través de la utilització de ferramentes ja presents a la vida diària (ordinador i mòbil) els alumnes s'aproximen a les solucions matemàtiques deduïdes de forma teòrica.

Es presenta la física tal com és, una rama de la ciència viva, en la qual comprovar les solucions teòriques té un caràcter bàsic i fonamental. El moviment harmònic simple passa a formar part de l'entorn, podent ser trobat en multitud de situacions diferents. Com va declarar una alumne: "Per tant, el vaivé del seient del conductor del bus es podria aproximar al de la peça penjada a la molla".

Tenim al nostre abast dues ferramentes amb grans possibilitats. El principal avantatge de l'aplicació "Physics Toolbox" és la visualització immediata del moviment. Per contra, el programa "Tracker" necessita un tractament del vídeo però ens retorna un major nombre de variables i un estudi més profund. A més, el domini del programa "Tracker" ens serà 
útil per l'estudi d'altres experiències com poden ser plans inclinats, caiguda lliure, entre d'altres.

\section{REFERÈNCIES}

BURBANO, S.; BURBANO, E; GRACIA, C (2003). Física General. Edición 32. Madrid: Editorial Tébar, S. L.
TIPLER, P. MOSCA, G. (2004). Física para la ciencia y la tecnología: Mecánica. Oscilaciones y Ondas. Termodinámica: 1. Edición 5. Barcelona: Editorial Rerverté.

CASELLAS, T. (2013).Smartphones...smartphysica. Recursos de Física, 12, 3/8.

LÓPEZ, V. (2015). L'acceleròmetre del mòbil. Una alternativa al sensor de distància? Recursos de Física, 15, 5/8. 